\title{
"Independent" reanalysis of landmark starch solutions trial was published by original authors
}

Clarification - This News article (BMJ 2017;358:j3552, doi:10. 1136/bmj.j3552) stated that the Duke Clinical Research Institute (DCRI) team that conducted the reanalysis was unsure about whether the contract between DCRI and the George Institute for Global Health, which commissioned the reanalysis, permitted
DCRI to publish independently of the George Institute. Since publication of this News article, Karen Pieper, who led the DCRI reanalysis, has clarified that the contract does grant DCRI the right to publish the results of the reanalysis. 